\title{
Liquid Biopsies to Evaluate Immunogenicity of Gynecological/Breast Tumors: On the Way to Blood-Based Biomarkers for Immunotherapies
}

\author{
Corinna Keup Rainer Kimmig Sabine Kasimir-Bauer \\ Department of Gynecology and Obstetrics, University Hospital Essen, Essen, Germany
}

\section{Keywords}

Liquid biopsy · Immuno-oncology · Immunotherapy · Immune checkpoints · Immune checkpoint inhibitors . Breast cancer - Gynecological cancer

\begin{abstract}
Background: Despite the assumption of breast cancer (BC) as a cold, non-immunogenic tumor, immune checkpoint inhibitor (ICI) therapy is favorable for a subgroup of patients. Immunohistochemical assessment of the programmed cell death ligand 1 (PD-L1) is the only approved companion diagnostic for anti-PD-L1 therapy in metastatic triple-negative BC; however, challenges regarding the standardization of PD-L1 scoring in tumor tissue still remain. Consequently, to select patients most likely to respond to $\mathrm{ICl}$, blood-based biomarkers are urgently needed. Summary and Key Messages: Liquid biopsy, comprising circulating immune cells, circulating tumor cells and extracellular vesicles, as well as their surface proteins, is of high potential, and these analytes were already shown to be molecular correlates or regulators of the evasion from antitumoral immune reaction. Liquid biopsy, also enabling the evaluation of tumor mutational burden (TMB), microsatellite instability, and the T-cell receptor repertoire, allows serial sampling for monitoring purposes and reflects intra-tumoral heterogeneity which qualifies as marker for immunogenicity. Only a very few studies have already elucidated the potential of these analytes as biomarkers under $\mathrm{ICI}$ therapy. Nonetheless, the topic is of growing interest and has high relevance for the future. However, for clinical implementation, these multifarious analytes first need to pass robust standardization and validation procedures.
\end{abstract}

C 2020 S. Karger AG, Basel

\section{Introduction}

Evasion from antitumoral immune responses is one hallmark of cancer [1]. Programmed cell death 1/programmed cell death ligand 1 (PD-1/PD-L1), as well as cytotoxic $\mathrm{T}$ lymphocyte-associated protein 4 (CTLA-4) are important immune checkpoint components that regulate the function of lymphocytes. More specifically, interaction of PD-1 with PD-L1 or CTLA- 4 with its binding partners leads to inhibition of T-cell function. Thus, immune checkpoint inhibitor (ICI) therapy with anti-PD-1/ PD-L1/CTLA-4 drugs is emerging as a new treatment modality in various cancer types and finally, was approved for several agents by the Food and Drug Administration (FDA) and the European Medicines Agency (EMA) [2]. However, besides a few exceptions, breast and gynecological cancers remain tumors that have not experienced high benefit from immunotherapy yet [3-5].

Breast cancer (BC) has been regarded as poorly immunogenic, and phase I and II studies with ICI in BC overall showed disappointing response rates [6-17], but revealed ICI to be successful in some BC subtypes like triple-negative (TNBC; ER/PR/HER2-negative) and HER2-positive BC [18]. The IMpassion 130 trial was the first phase III study to show benefit of ICI in BC $[19,20]$ and resulted in the approval of the PD-L1 inhibitor atezolizumab in combination with nab-paclitaxel for patients with unresectable locally advanced or metastatic TNBC whose tumors showed PD-L1 expression [21]. In April 2020, 143 interventional clinical trials were registered to elucidate the benefit of further ICIs, in other BC subgroups and in diverse combinational regimens [22]. 


\begin{tabular}{|c|c|c|c|}
\hline \multicolumn{4}{|c|}{ Abbreviations used in this paper } \\
\hline $2 \mathrm{~B} 4$ & cluster of differentiation 244 & IL-4 & interleukin 4 \\
\hline $\mathrm{BC}$ & breast cancer & ILT2 & leukocyte immunoglobulin-like receptor subfamily \\
\hline $\mathrm{BRCA} 1 / 2$ & breast cancer type $1 / 2$ susceptibility protein & & B member 1 \\
\hline CAMLs & cancer associated macrophage-like cells & $\mathrm{JAK} 1 / 2$ & janus kinase $1 / 2$ \\
\hline CD127 & interleukin-7 receptor subunit alpha & LAG3 & lymphocyte-activation gene 3 \\
\hline CD14 & cluster of differentiation 14 & LB & liquid biopsy \\
\hline $\mathrm{CD} 25$ & interleukin-2 receptor alpha chain & $\mathrm{Mbp}$ & mega base pair \\
\hline CD28 & cluster of differentiation 28 & MHC & major histocompatibilty complex \\
\hline CD4 & cluster of differentiation 4 & MSI & microsatellite instability \\
\hline CD40 & cluster of differentiation 40 & NK & natural killer \\
\hline CD47 & cluster of differentiation 47 & OC & ovarian cancer \\
\hline CD8 & cluster of differentiation 8 & OS & overall survival \\
\hline CD80 & cluster of differentiation 80 & PARP & poly(ADP-Ribose)-Polymerase 1 \\
\hline cfDNA & cell-free DNA & PBMCs & peripheral blood mononuclear cells \\
\hline CTCs & circulating tumor cells & PD-1 & programmed cell death 1 \\
\hline ctDNA & circulating tumor DNA & PD-L1 & programmed cell death ligand 1 \\
\hline CTLA-4 & cytotoxic T lymphocyte-associated protein 4 & PD-L2 & programmed cell death ligand 2 \\
\hline CXCR4 & $\mathrm{C}-\mathrm{X}-\mathrm{C}$ chemokine receptor type 4 & PFS & progression free survival \\
\hline EMA & European Medicines Agency & PIK3CA & phosphatidylinositol-4,5-bisphosphate 3-kinase, \\
\hline EMT & epithelial to mesenchymal transition & & catalytic subunit alpha \\
\hline ER & estrogen receptor & $\mathrm{PR}$ & progesteron receptor \\
\hline EVs & extracellular vesicles & PTEN & phosphatase and tensin homolog \\
\hline FasL & Fas ligand & S & soluble \\
\hline FDA & Food and Drug Administration & STAT3 & signal transducer and activator of transcription 3 \\
\hline FOXP3 & forkhead box P3 & TAMs & tumor associated macrophages \\
\hline GITR & glucocorticoid-induced TNFR-related protein & TCR & T cell receptor \\
\hline HER2 & human epidermal growth factor receptor 2 & TIGIT & T cell immunoreceptor with Ig and ITIM domains \\
\hline HLA & human leukocyte antigens & TILs & tumor-infiltrating lymphocytes \\
\hline ICI & immune checkpoint inhibitor & TIM-3 & hepatitis A virus cellular receptor 2 \\
\hline ICOS & inducible T-cell costimulatory, CD278 & TMB & tumor mutational burden \\
\hline IDO & indoleamine-pyrrole 2,3-dioxygenase & TME & tumor microenviroment \\
\hline IFN $\gamma$ & interferon gamma & TNBC & triple-negative \\
\hline $\mathrm{IHC}$ & immunohistochemistry & TNFa & tumor necrosis factor alpha \\
\hline IL-10 & interleukin 10 & TP53 & tumor protein p53 \\
\hline IL-2 & interleukin 2 & VISTA & $\mathrm{V}$-domain Ig suppressor of $\mathrm{T}$ cell activation \\
\hline
\end{tabular}

In ovarian cancer (OC), the use of antibodies inhibiting CTLA-4, PD-1 or PD-L1 resulted in response rates of only $10-15 \%$. The combination of anti-PD-1 nivolumab and anti-CTLA-4 ipilimumab was more promising in platinum-resistant OC with a 6-month overall response rate of $34 \%$. However, up to now, data are too preliminary to obtain approval from the FDA [5]. For cervical cancer, pembrolizumab monotherapy demonstrated durable antitumor activity and manageable safety in patients with advanced disease which granted accelerated approval of pembrolizumab by the FDA for patients with advanced PD-L1-positive tumors who experienced progression during or after chemotherapy [3].

Currently, PD-L1 expression is assessed by immunohistochemistry (IHC) for stratifying patients to receive ICI [21]. However, there are some limitations for the evaluation of PD-L1 expression in tumor tissue. Consequently, in the view of toxicity profiles of these therapies and immense costs, it has been hypothesized that the use of body fluids, the so-called liquid biopsy (LB), including circulating tumor cells (CTCs), cell-free DNA (cfDNA)/ circulating tumor DNA (ctDNA), microRNAs as well as extracellular vesicles (EVs) could be an ideal "surrogate tissue" to represent predictive markers more appropriately [2]. However, LB is not implemented yet in clinical routine for ICI response prediction [2].

This review elucidates the complexity of LB in gynecological cancers and especially in $\mathrm{BC}$ as source of potential markers to evaluate immunogenicity and stratify patients for immunotherapies.

\section{Need for Preferentially Blood-Based Biomarker}

Responses to ICI are variable in gynecological/breast tumor patients, and resistance is common $[23,24]$. The assumed poor immunogenicity of $\mathrm{BC}$ is thought to cause these low response rates. Differentiation of cold (non-immunogenic) and hot (immunogenic) tumors highlights the need for biomarkers to select for predispositions to 
trigger an immune response [25]. The identification of biomarkers is dependent on understanding the complex interaction between tumor and immune cells, and combinational therapies might be proposed to prime cold, nonimmunogenic tumors to increased immunogenicity, as thought to be possible by chemotherapy inducing antigen release [26] and by PARP inhibitors inducing higher mutational burden and activating PD-L1 expression [27, 28].

It has already been shown that the lines of treatment are predictive for the response of BC patients to ICI [29] and due to the fact that HER2-positive and TNBC patients showed an increased benefit from ICI as compared to other BC subgroups $[6,15]$, IHC detecting the hormonal receptors and HER2 in tumor tissue might be used as first hint for ICI response prediction. In addition, IHC of PD-L1 expressed by tumor-infiltrating lymphocytes (TILs) is the only FDA-approved test to stratify metastatic TNBC patients to receive atezolizumab plus nab-paclitaxel [21].

However, the routine diagnostic for gynecological and breast tumors does not reveal the complex intra-tumoral heterogeneity and complex systemic tumor biology. For example, the immune surveillance may differ between primary tumor and metastatic sites [30], as recently shown in a preliminary data set for the expression of the immune checkpoints IDO1 and PD-L1 in matched primary tumor tissue and metastases from TNBC patients [31]. Tumor tissue biopsy is suitable to evaluate the interactions and characteristics of tumor cells and immune cells once, but serial assessment of the tumor microenvironment through tissue biopsies is not feasible and, consequently, ICI response monitoring is not possible via tissue biopsies.

In contrast, $\mathrm{LB}$ is suitable for serial therapy monitoring and reflects the tumoral heterogeneity. Besides CTCs, cfDNA, ctDNA and EVs, classically evaluated as LB sources in blood samples of cancer patients, circulating immune cells and circulating peptides (such as immune checkpoints, chemokines, and cytokines) might be relevant as blood-based biomarkers for ICI therapy management as well (Fig. 1). Importantly, immune cells, CTCs, EVs and proteins in blood might be regulators of ICI response and can simultaneously be used as biomarkers. There are already hints in the literature that most of the genomic markers presented here might be evaluable in cfDNA and CTCs in a parallel analysis [32].

\section{Immune Cells and Related Proteins}

\section{Circulating Immune Cells}

The clinically available ICIs activate cytotoxic T cells and modulate regulatory $\mathrm{T}$ cells. Both cell types can be isolated from blood via density centrifugation and/or se- lection of cells with specific surface proteins by flow cytometry [33]. In TNBC patients, it has already been shown that the fraction of CD25+ FOXP3+ cells in circulating CD4+ T cells was positively correlated with the level of cells showing the same characteristics in the tumor tissue. In contrast, the level of CD25+ FOXP3- cells in circulating CD4+ T cells was not associated with the level of cells harboring equivalent surface marker expression in the tumor tissue [34]. The number of circulating CD4+ CD25+ CD127- FOXP3 + cells was elevated in BC patients experiencing tumor progression [35]. Most recently, the preliminary data of the IMPRIME1 study suggest the increase in activated monocytes (defined by CD86+) and increase in activated $\mathrm{T}$ cells (defined by PD-1+/HLA$\mathrm{DR}+/ \mathrm{KI} 67+$ ) evaluated by flow cytometry after 3 weeks of treatment with pembrolizumab plus a novel innate immune activator in the blood of metastatic TNBCs to be associated with increased progression-free survival (PFS) and overall survival (OS) [36].

Circulating immune cells can be characterized by gene expression profiling of peripheral blood mononuclear cells (PBMCs). Comparison of the immune checkpoint gene expression in matched tissue and blood revealed an upregulation of CTLA-4, TIM-3, TIGIT and PD-L1 in BC PBMCs, that was explained by an aberrant promoter methylation profile [37]. In contrast to healthy donors, mRNA expression of FOXP3, CTLA-4, and GITR was significantly higher in PBMCs of $B C$ patients [38], and the mRNA expression of the major immune regulators $P D$ L1, FOXP3, CD80, CD40, and CD14 in PBMCs was associated with $B C$ progression [39].

Importantly, Gao et al. [40] reported at the San Antonio Breast Cancer Symposium 2019 that BC patients responding to anti-PD-1 treatment with pembrolizumab exhibited a lower CTLA- 4 expression in circulating CD4+ $\mathrm{T}$ cells at baseline compared to non-responders. Additionally, a lower percentage of CD4+ T cells expressing the exhaustion markers CTLA-4, TIM-3 and 2B4 at baseline was a favorable factor for pembrolizumab therapy of BC patients [40].

\section{Human Leukocyte Antigens}

Human leukocyte antigens (HLA) have the ability to induce apoptosis in CD8+ T cells and natural killer cells via the Fas ligand pathway [41]. It has already been shown that the expression of HLA-G molecules is a tumor immune evasion mechanism. Detection of HLA-G bound to EVs in blood was first shown in BC patients by $\mathrm{Yu}$ et al. [42] and later HLA-G bound to EVs detected in blood of primary $\mathrm{BC}$ patients before neoadjuvant chemotherapy was demonstrated to be associated with progression and the presence of stem-cell like CTCs [43]. This finding was contrasted by the association of soluble HLA-G, not bound to EVs, in the same cohort with improved out- 
Fig. 1. Liquid biopsy analytes to evaluate breast cancer immunogenicity. Circulating tumor cells (CTCs, orange), extracellular vesicles (EVs, yellow), and cell-free DNA (cfDNA) or cell-free tumor DNA (ctDNA), as well as T cells (blue) and circulating peptides have the potential as blood-based biomarker to predict response to immune checkpoint inhibitor therapy in breast cancer patients. The diversity of T-cell receptors (TCRs, green) is illustrated by the different shades of green. 2B4, cluster of differentiation 244; CD28, cluster of differentiation 28; CD47, cluster of differentiation 47; cfDNA, cell-free DNA; CTC, circulating tumor cell; ctDNA, cell-free tumor DNA; CTLA-4, cytotoxic T lymphocyte-associated protein 4 ; CXCR4, C-X-C chemokine receptor type 4; EVs, extracellular vesicles; HLA, human leukocyte antigens; IFN- $\gamma$, interferon- $\gamma$; IL-10, interleukin-10; IL-2, interleukin-2; IL-4, interleukin-4; methyl-C, methylated cytosine; MHC, major histocompatibility complex; MSI, microsatellite instability; PD-1, programmed cell death $1 ; \mathrm{PD}-\mathrm{L} 1$, programmed cell death ligand 1; PD-L2, programmed cell death ligand 2; TCR, T-cell receptor; TIM-3, hepatitis A virus cellular receptor 2; TMB, tumor mutational burden; unmethyl-C, unmethylated cytosine.

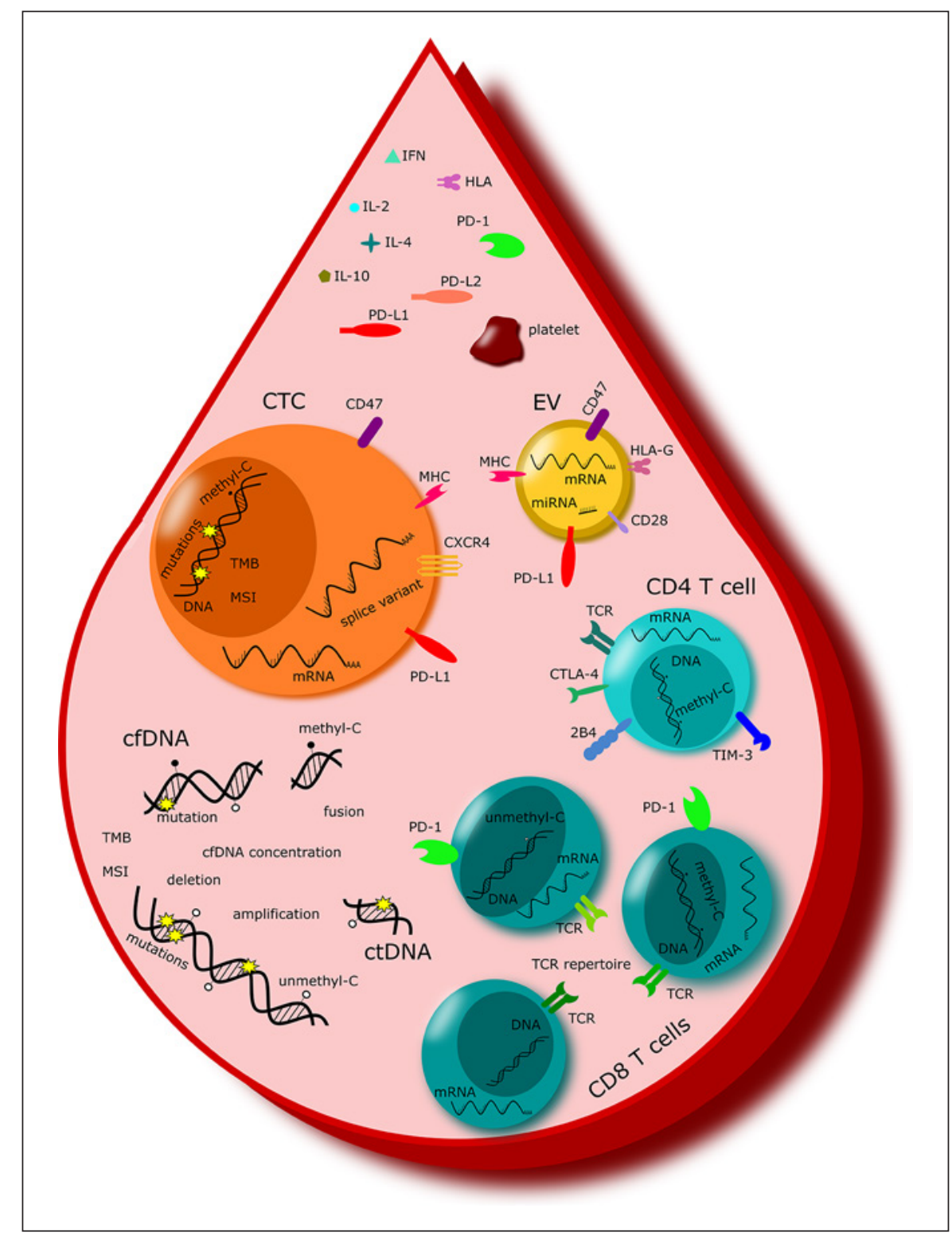

come [43], highlighting the complex interplay of the diverse structural HLA-G variants with their binding partner ILT2 [44]. Similarly, vesicle-bound HLA-G was associated with residual tumor, presence of CTCs, and disease progression in epithelial OC patients [45]. However, since the level of classical HLA molecules (HLA-A, -B, $-C)$ is significantly higher in serum than the level of nonclassical HLA molecules (HLA-G), the immunosuppressive effects might mainly be due to classical HLA molecules [41]. Probably, HLA loss might be a predictive marker for ICI therapy in BC, as described for melanoma and NSCLC patients [46].

\section{Programmed Death Ligand 1}

PD-L1, also known as CD247 or B7-H1, is expressed on dendritic and tumor cells. Binding of PD-L1 to PD-1 (also known as PDCD1, CD279, SLEB2, hPD-1, hPD-1, and hSLE1 and expressed on T cells) inhibits T-cell activation and thus, leads to impaired antitumoral immune response. Anti-PD-L1 therapy demonstrated good response rates in patients with high expression of PD-L1 assessed by IHC on tumor tissues, but some patients with low PD-L1 expression also indicated a benefit from ICI [25]. This discrepancy was explained by the dynamic nature of immune modulation and cannot be adequately represented by a static snapshot. Furthermore, PD-L1 is expressed in tumor and immune cells complicating the assessment of PD-L1 protein expression in the tumor tissue biopsy. In addition, the variable detection antibodies remain an unsolved issue for standardization of PD-L1 assessment. Finally, the heterogenous expression between primary tumor and metastatic tissue also limits the use of IHC [47, 48]. Blood-based assessment of PD-L1 is conceivable by protein, transcript, mutation, or methyla- 
tion analysis of CTCs, EVs, circulating immune cells, and circulating nucleic acids.

\section{In PBMCs}

Evaluation of PMBCs from BC patients demonstrated that $\mathrm{PD}-\mathrm{L} 1$ promotor hypomethylation might be the reason for increased PD-L1 expression in PBMCs compared to matched tumor tissue [37]. Furthermore, the PD-1 promotor was shown to be hypermethylated in PBMCs compared to tumor tissue [37]. Methylation profiling via cfDNA might consequently be considered as molecular correlate for PD-L1 expression. Another study revealed that the number of PD-1 high CD8+ T cells in tumor tissue was significantly greater compared to matched blood of TNBC patients. The PD-1 high CD $8+$ T cells showed a skewed T-cell receptor (TCR) repertoire and characteristics of a state of exhaustion [49]. These studies detail the differences between tumor immune interactions in tumor tissue and in circulation.

PD-L1 gene amplification might also be evaluated in cfDNA, while PD-L1 expression was detected in cfRNA in the majority of BC patients' blood [50].

\section{On CTCs}

Whereas a variety of studies have analyzed PD-L1 on CTCs in lung cancer, only a few studies have addressed other solid tumors [2]. For BC, only two studies have already analyzed PD-L1 on CTCs. In hormone receptor-positive, HER2-negative metastatic BC patients using the EpCAMbased CellSearch system as well as western blotting and flow cytometry, PD-L1-positive CTCs at different expression levels were detected in $11 / 16$ patients [51]. This high prevalence of $\mathrm{PD}$-L1-positive $\mathrm{BC}$ patients contrasted with the knowledge that $\mathrm{BC}$ cells have low PD-L1 expression and was explained by a possibly higher PD-L1 expression by CTCs than by primary tumors due to the continuous contact of CTCs with T lymphocytes, activating interferon- $\gamma$ $($ IFN- $\gamma)$ secretion, which leads to an adaptive increase in PD-L1 expression on the tumor cells [52].

However, these findings are comparable with results obtained for lung cancer $[53,54]$ and were also confirmed in a recently published BC paper by Papadaki et al. [55]. In this more comprehensive BC analysis, PDL1 and CD47 expression (the "do not eat me" signal on hematopoietic blood cells, often adopted by tumor cells to escape killing from macrophages) was investigated on PBMC cytospins from 100 early and 98 metastatic BC patients, by triple immunofluorescence for CD47/ PD-L1/cytokeratins. A low concordance between PDL1 and CD47 marker expression on CTCs and tumor tissue as well as PBMCs and TILs was demonstrated. CD47+ and/or PD-L1+ CTCs were detected in about 11,17 , and $30 \%$ of early, recurrent, and de novo metastatic patients, respectively. In metastatic disease, the presence of CD47 high and/or PD-L1 high CTCs was associated with disease progression, shorter PFS and was an independent predictor for an increased risk of relapse and death [55].

Although these data are promising, further research is necessary to assess the clinical usefulness of PD-L1 expression by CTCs for entry into clinical studies. There are still open questions that must be answered before using this LB marker for treatment decisions. In this regard, there is evidence for a bidirectional cross talk between PD-L1 expression and epithelial to mesenchymal transition $[56,57]$. Methodological issues are of high importance to prevent false-positive results since PD-L1 is also expressed on myeloid-derived suppressor cells, as well as on tumor-associated macrophages in OC and on cancer-associated macrophage-like cells in lung cancer [58-60].

\section{On EVs}

In other tumor entities, PD-L1 was detected bound to EVs circulating in blood [61-63]. Importantly, in BC cell culture experiments, vesicle-bound PD-L1 was shown to be able to bind to PD-1, inhibiting T-cell activation, suppressing $\mathrm{T}$-cell killing of $\mathrm{BC}$ cells and promoting tumor growth [64]. Moreover, EVs were shown to transport PDL1 from PD-L1-positive to PD-L1-negative BC cells [64] and consequently, EVs might spread this cellular immune evasion tool.

\section{Soluble}

In breast and gynecological cancers, a variety of studies have also analyzed plasma samples as one further LB source for the detection of PD-1, PD-L1, PD-L2, and other proteins. In a cohort of 66 TNBC patients, serum soluble (s) PD-1 levels were significantly elevated before neoadjuvant chemotherapy; however, only an increased tendency for sPD-L1 levels was observed when compared with the results of 59 healthy women. sPD-1 and sPD-L1 before therapy increased with tumor stage, and patients who experienced complete or partial remission under neoadjuvant chemotherapy had significantly decreased serum levels of sPD-1 and sPD-L1 as compared to patients with a poor response to therapy [65]. In OC, it was recently demonstrated that sPD-L1 was significantly increased as compared to controls and was associated with residual tumor burden, a reduced 5-year OS and reduced PFS. Strikingly, sPD-L1 levels $>6.4 \mathrm{pg} / \mathrm{mL}$ were indicative of a reduced OS and PFS in platinumsensitive patients, while OS and PFS in platinum-resistant patients did not differ when patients were stratified according to this cut-off. In contrast, sPD-L2 was significantly decreased and was associated with platinumresistance and the presence of CTCs expressing markers for resistance [66]. 


\section{Circulating Peptides}

Besides the detection of HLA molecules and PD-L1 in plasma of BC patients, increased levels of IL- 4 and IL-10, but decreased levels of IFN- $\gamma$ and IL-2, were found in BC patients progressing under therapy [35] and it might be hypothesized that IFN- $\gamma$ and IL-2 levels in plasma can be positive predictive markers for ICI response, while IL-4 and IL-10 levels might show resistance to ICI. Immunerelated peptides conceivable in plasma could also include the immune checkpoints B4-H7, ICOS, VISTA, IDO, or LAG3. This assessment is known as functional analysis and provides quantitative information about type and quality of immune response.

\section{Genomic Markers}

\section{TCR Repertoire}

In general, the clinical activity of ICI was correlated with the T-cell recognition of neoantigens. These neoantigens, generated by non-synonymous de novo somatic mutations, are presented to T cells via major histocompatibility complex (MHC) molecules and are recognized as non-self if corresponding TCRs exist [67]. In general, the TCR consists of one $\alpha$ - and one $\beta$-chain, each containing three hyper-variable regions. One of these regions, CD3, is a product of recombination and unique to each TCR [68]. Sequencing of the CD3 region of TCRs elucidates the TCR repertoire [69], that is also known as TCR diversity and frequently discussed as biomarker for ICI response [70]. Narrowing the TCR repertoire is called TCR convergence and is often described after ICI treatment [71].

The changing TCR repertoire under chemotherapy and a correlation of TCR repertoire with clinical responses was shown in circulating CD8+ T cells in BC patients' blood, where an increased TCR repertoire was associated with better outcome [72]. TCR sequencing in BC tumor tissue and matched blood displayed no correlation between intra-tumoral and peripheral blood TCR repertoire [73]. While TCRs were more polyclonal in blood, most of the expanding clones in tumor tissue were not detectable in blood [73].

TCR sequencing from blood has already been employed in BC patients receiving ICI. In a small cohort, blood-based TCR sequencing from PBMCs of 6 patients with metastatic inflammatory BC and 9 patients with metastatic TNBC revealed that a lower TCR repertoire in combination with low CD4+ T-cell exhaustion markers showed a trend towards ICI response and longer progression-free interval [40]. In summary, TCR sequencing can be employed as indirect quantification for T cells, can elucidate the TCR diversity and the development of the TCR repertoire and $\mathrm{T}$-cell expansion over time. All these factors seem to have potential as ICI response biomarkers in BC.

\section{Tumor Mutational Burden}

The tumor mutational burden (TMB), as indirect measurement of T-cell activation by neoantigens, is currently discussed as biomarker for ICI response [74], however, companion diagnostic approval for TMB has been withdrawn from the FDA and the approval from the EMA is still ongoing [2]. For TMB determination, a large set of genes, typically more than 300 , is sequenced and the number of non-synonymous mutations per mega base pair $(\mathrm{Mbp})$ is stated. In contrast to the orthogonal marker PD-L1, TMB is objective and quantifiable [75], but germline mutations have to be excluded and mutations acquired by clonal hematopoiesis of indeterminate potential might cause artefacts in TMB measurement. High TMB is mostly referred to as $>10$ mutations/ Mbp, but the determined TMB greatly differs between targeted panel sequencing (with $>300$ genes) and wholeexome sequencing, so the TMB scoring has to be adapted based on the sequencing method $[76,77]$. Most studies elucidating TMB as ICI biomarker use tumor tissue biopsies, but cfDNA and probably also CTCs, can be suitable LB sources for serial TMB assessment. Employing chromatin immunoprecipitation sequencing, the variants of tumor-derived cfDNA (ctDNA) and variants of cfDNA, released by non-pathogenic cells, might even be differentiated [78].

The potential of blood TMB has already been shown for ICI response prediction in non-small cell lung cancer and melanoma [79-81]. In metastatic TNBC patients, however, not blood-based TMB measurement but high tissue TMB (>10 non-synonymous mutations per Mbp; in $18 \%$ of cases) was significantly associated with longer PFS under ICI $[82,83]$. Nonetheless, the prevalence of high TMB in $\mathrm{BC}$ is low, $3 \%$ in primary tumors and $8 \%$ in metastatic tumors [82], and a correlation of high tissue TMB with ICI response has not been confirmed in all available BC studies [84].

Fusions [85] as well as deficiencies in the splicing machinery [86] also induce neoantigen release and their detection in cfDNA might be an alternative indirect correlate for immunogenicity in plasma. In contrast to the quantitative assessment of mutant fragments in plasma as blood TMB, the level of cfDNA itself was proposed as early monitoring marker under ICI treatment, since the dynamic changes in cfDNA quantities might predict long-term outcome $[87,88]$.

\section{Microsatellite Instability}

In relation to $\mathrm{TMB}$, general genomic instability can also be measured by the number of variations within the microsatellite loci, called microsatellite instability (MSI). MSI assessment can be conducted with targeted panels including $>300$ genes or genome-wide sequencing, both usable for TMB assessment as well, but for MSI analysis 
only low coverage is needed. cfDNA is increasingly used as LB source to evaluate the genomic instability in blood and it was shown in cfDNA of patients with solid tumors, including $\mathrm{BC}$, that the decrease in genomic instability under ICI correctly predicted progression and identified cases of pseudo-progression $[89,90]$. As the entirety of cfDNA includes DNA of non-pathogenic cells, evaluation of the genomic instability in genomic DNA of CTCs might be useful as well.

\section{Specific Mutations}

DNA repair deficiency, in BC commonly known via $B R C A 1 / 2$ mutations, can cause genomic instability and high TMB, which both are ICI response markers as discussed above. Consequently, it was speculated whether $B R C A 1 / 2$ mutant BCs might benefit from ICI. In this context, it was shown that BRCA1 mutant TNBCs had a higher tissue TMB, more TILs, and a higher expression of $\mathrm{PD}$ L1 and CTLA-4 as compared to wild-type TNBCs [91]. However, in hereditary BRCA1/2 mutant $\mathrm{BC}$ and $\mathrm{OC}$ patients, TMB was not associated with the magnitude of immune response [92]. The promising effect of ICI in mismatch repair-deficient BCs might be due to the combinational regimen with PARP inhibitors [27], which were shown to induce PD-L1 expression [28, 93]. BRCA mutations can be assessed by using cfDNA or genomic DNA of CTCs $[94,95]$ as potential LB sources for immunogenicity.

In addition, other specific mutations might be used as predictive markers and can be assessed in the future via cfDNA or genomic DNA of CTCs. Genomic defects in the IFN- $\gamma$ pathway genes, like $J A K 1 / 2$, are frequently discussed to cause resistance to ICI $[24,52]$ and were identified in non-responders under ICI [23]. In metastatic TNBC, genomic PTEN alterations were associated with limited clinical benefit of ICI, shorter PFS, and shorter OS [83]. Variants in TP53 were associated with immunogenic activity in TNBCs in the past [96] and, consequently, the TP53 mutant, but PIK3CA wild-type, TNBC genotype was demonstrated to be a favorable marker for ICI treatment [97].

\section{Circulating Tumor Cells}

A variety of technologies have been developed for CTC enrichment and detection in the past. CTC isolation and/ or enrichment is mainly based on positive immunomagnetic selection (often EpCAM-based) or is size-based, while negative immunomagnetic selection for the elimination of unwanted white blood cells has also been used [98]. CTC detection uses immunocytochemistry, usually applied for counting the number of CTCs in $7.5 \mathrm{~mL}$ of blood (FDA-approved CellSearch ${ }^{\circledR}$ system, Menarini, Silicon Biosystems) or molecular analysis for getting deeper insights into the genome, transcriptome, and proteome of these cells [99].

CTCs closely interact with circulating immune cells, such as neutrophils and macrophages, to survive in the blood stream [100], and extravasion of tumor cells into the circulation is supported by immune cells. BC patients who harbored CTCs exhibited more intra-tumoral and peri-tumoral regulatory $\mathrm{T}$ cells than $\mathrm{BC}$ patients with no CTCs [101]. Mego et al. [102] demonstrated an inverse correlation of CD8+ T cells in tumor tissue and CTC amount in $\mathrm{BC}$ patients, as well as an inverse correlation of CTC count and the number of myeloid dendritic cells capable of producing TNF- $\alpha$ in patients with inflammatory BC. In a more recent study presented at the San Antonio Breast Cancer Symposium 2019, they demonstrated a correlation of CTCs in epithelial to mesenchymal transition with an increased PD-L1 expression in the tumor-associated stroma of early BC patients [103]. Thus, CTCs might be an LB correlate of the immunogenic situation in tumor tissue.

Moreover, the properties of CTCs might also be relevant to evaluate immunogenicity. Besides the analysis of PD-L1 and CD47 on BC CTCs [51, 55], as described above, the $\mathrm{C}-\mathrm{X}-\mathrm{C}$ chemokine receptor type 4 , regulating the function and trafficking of immune cells, was already found on BC CTCs [104]. New sophisticated technologies enable functional analysis of even single CTCs, not only on the proteomic level, but also on the transcriptomic and metabolic level and might reveal more potential ICI markers in the future.

\section{Extracellular Vesicles}

EVs are secreted by pathogenic and non-pathogenic cells and present an intercellular communication strategy. EVs were frequently shown to be involved in the interplay of tumor cells and immune cells. Tumor-derived EVs were mostly shown to have immunosuppressive features, supporting the immune evasion mechanisms of tumors [105] via downregulation of MHCII in dendritic cells [106], activation of the cGAS-STING signaling in dendritic cells [107], induction of STAT3-mediated M2 polarization in monocytes $[108,109]$, downregulation of IFN- $\gamma$ expression in natural killer cells [110], and apoptosis of T cells [111]. The presentation of PD-L1 on the EV surface, detailed above, promoted T-cell exhaustion and suppression of antitumoral immune activity [61, 112]. In BC patients, the "do not eat me signal" $C D 47$, detected on BC CTCs [55], was also detected on BC EVs, in contrast to healthy donors [113], and correlated with BC status [114].

Mechanisms of immune activation by stimulation of natural killer cells, macrophages, and B- and T-cells were 
also described to be mediated by tumor-derived EVs [115, 116]. Immune activation resulting in robust tumor clearance was associated with EVs affecting the Hippo pathway [117]. EVs derived from antigen-presenting cells were frequently shown to have immunostimulatory properties by carrying MHC complexes to induce T-cell activation [118, 119], although T-cell stimulation by EVs was less effective than that by antigen-presenting cells [120]. Monocyte-derived EVs can also stimulate CD8+ T cells $[121,122]$.

With regard to EVs as markers for immuno-oncology, an increase in vesicular PD-L1 in melanoma patients under ICI indicated adaptive response, stratified clinical responders from non-responders [61], and increasing vesicular PD-L1 and CD28 levels were associated with improved PFS and OS in patients under anti-CTLA-4 therapy [123]. Even vesicular RNA profiles were detected to correlate with response to anti-CTLA- 4 therapy in these patients [124].

In summary, EVs function as major immune regulators, and profiling of circulating EV s has high potential as marker for immunogenicity. Profiling of EVs circulating in BC patients' blood is common [125-129] but major issues remain regarding a validated and standardized isolation procedure, which have to be solved before the clinical implementation of EVs is attainable [115].

\section{Conclusion}

LB is a source for multifarious potential biomarkers to evaluate immunogenicity. Despite remaining challenges concerning isolation, detection, standardization, and validation, CTCs and EVs might be of high relevance regarding ICI response prediction. Emerging liquid biomarkers, namely platelets, circulating immune cells, and circulating peptides might be interesting to be further investigated with regard to their usefulness in immuno-oncology. At the moment, the greatest potential is seen in the genomic markers MSI, TCR repertoire, and specific mutations, for which promising data have been published with regard to blood analysis in BC under ICI.

\section{Conflict of Interest Statement}

C.K. received support from QIAGEN to cover travel expenses. R.K. has received honoraria/is part of the advisory board from/at Tesaro, Astra-Zeneca, and Medtronic in the last 3 years. R.K. is council of IGCS, president of SERGS and proctored and presented for Intuitive Surgical. S.K.-B. is a consultant for QIAGEN and has received honoraria from Novartis.

\section{Funding Sources}

There are no funding sources to declare.

\section{Author Contributions}

C.K. and S.K.-B. did the literature research and wrote and revised the manuscript. R.K. revised the manuscript. C.K. created Figure 1.

\section{References}

1 Hanahan D, Weinberg RA. Hallmarks of cancer: the next generation. Cell. 2011 Mar; 144(5):646-74.

2 Hofman P, Heeke S, Alix-Panabières C, Pantel K. Liquid biopsy in the era of immunooncology: is it ready for prime-time use for cancer patients? Ann Oncol. 2019 Sep;30(9): 1448-59.

3 Chung HC, Ros W, Delord JP, Perets R, Italiano A, Shapira-Frommer R, et al. Efficacy and Safety of Pembrolizumab in Previously Treated Advanced Cervical Cancer: Results From the Phase II KEYNOTE-158 Study. J Clin Oncol. 2019 Jun;37(17):1470-8.

4 Planes-Laine G, Rochigneux P, Bertucci F, Chrétien AS, Viens P, Sabatier R, et al. PD-1/ PD-L1 Targeting in Breast Cancer: The First Clinical Evidences Are Emerging. A Literature Review. Cancers (Basel). 2019 Jul;11(7): 11.

5 Ghisoni E, Imbimbo M, Zimmermann S, Valabrega G. Ovarian Cancer Immunotherapy: turning up the Heat. Int J Mol Sci. 2019 Jun; 20(12):20.
6 Nanda R, Chow LQ, Dees EC, Berger R, Gupta S, Geva R, et al. Pembrolizumab in Patients With Advanced Triple-Negative Breast Cancer: Phase Ib KEYNOTE-012 Study. J Clin Oncol. 2016 Jul;34(21):2460-7.

7 Adams S, Schmid P, Rugo HS, Winer EP, Loirat D, Awada A, et al. Phase 2 study of pembrolizumab (pembro) monotherapy for previously treated metastatic triple-negative breast cancer (mTNBC): KEYNOTE-086 cohort A. J Clin Oncol. 2017;35 15_suppl:1008.

8 Vinayak S, Tolaney SM, Schwartzberg LS, Mita MM, McCann GA, Tan AR, et al. TOPACIO/Keynote-162: Niraparib + pembrolizumab in patients (pts) with metastatic triplenegative breast cancer (TNBC), a phase 2 trial. J Clin Oncol. 2018;36 15_suppl:1011.

9 Nanda R, Liu MC, Yau C, Shatsky R, Pusztai L, Wallace A, et al. Effect of Pembrolizumab Plus Neoadjuvant Chemotherapy on Pathologic Complete Response in Women With Early-Stage Breast Cancer: An Analysis of the Ongoing Phase 2 Adaptively Randomized ISPY2 Trial. JAMA Oncol. 2020 Feb;6(5):676.
10 Voorwerk L, Slagter M, Horlings HM, Sikorska K, van de Vijver KK, de Maaker M, et al. Immune induction strategies in metastatic triple-negative breast cancer to enhance the sensitivity to PD-1 blockade: the TONIC trial. Nat Med. 2019 Jun;25(6):920-8.

11 Loibl S, Untch M, Burchardi N, Huober J, Sinn BV, Blohmer JU, et al. A randomised phase II study investigating durvalumab in addition to an anthracycline taxane-based neoadjuvant therapy in early triple-negative breast cancer: clinical results and biomarker analysis of GeparNuevo study. Ann Oncol. 2019 Aug;30(8):1279-88.

12 Chia S, Bedard PL, Hilton J, Amir E, Gelmon K, Goodwin R, et al. A Phase Ib Trial of Durvalumab in Combination with Trastuzumab in HER2-Positive Metastatic Breast Cancer (CCTG IND.229). Oncologist. 2019 Nov; 24(11):1439-45. 
13 Zimmer AS, Nichols E, Cimino-Mathews A, Peer C, Cao L, Lee MJ, et al. A phase I study of the PD-L1 inhibitor, durvalumab, in combination with a PARP inhibitor, olaparib, and a VEGFR1-3 inhibitor, cediranib, in recurrent women's cancers with biomarker analyses. J Immunother Cancer. 2019 Jul;7(1):197.

14 Liu JF, Gordon M, Veneris J, Braiteh F, Balmanoukian A, Eder JP, et al. Safety, clinical activity and biomarker assessments of atezolizumab from a Phase I study in advanced/recurrent ovarian and uterine cancers. Gynecol Oncol. 2019 Aug;154(2):314-22.

15 Emens LA, Cruz C, Eder JP, Braiteh F, Chung C, Tolaney SM, et al. Long-term Clinical Outcomes and Biomarker Analyses of Atezolizumab Therapy for Patients With Metastatic Triple-Negative Breast Cancer: A Phase 1 Study. JAMA Oncol. 2019 Jan;5(1):74-82.

16 Dirix LY, Takacs I, Jerusalem G, Nikolinakos P, Arkenau HT, Forero-Torres A, et al. Avelumab, an anti-PD-L1 antibody, in patients with locally advanced or metastatic breast cancer: a phase $1 b$ JAVELIN Solid Tumor study. Breast Cancer Res Treat. 2018 Feb;167(3):671-86.

17 Bajor DL, Mick R, Riese MJ, Huang AC, Sullivan B, Richman LP, et al. Long-term outcomes of a phase I study of agonist CD40 antibody and CTLA-4 blockade in patients with metastatic melanoma. OncoImmunology. 2018 Aug;7(10):e1468956.

18 Bertucci F, Finetti P, Birnbaum D, Mamessier E. The PD1/PDL1 axis, a promising therapeutic target in aggressive breast cancers. OncoImmunology. 2015 Aug;5(3):e1085148.

19 Schmid P, Adams S, Rugo HS, Schneeweiss A, Barrios CH, Iwata H, et al.; IMpassion130 Trial Investigators. Atezolizumab and $\mathrm{Nab}-\mathrm{Pa}-$ clitaxel in Advanced Triple-Negative Breast Cancer. N Engl J Med. 2018 Nov;379(22): 2108-21.

20 Schmid P, Rugo HS, Adams S, Schneeweiss A Barrios CH, Iwata H, et al.; IMpassion130 Investigators. Atezolizumab plus nab-paclitaxel as first-line treatment for unresectable, locally advanced or metastatic triple-negative breast cancer (IMpassion130): updated efficacy results from a randomised, double-blind, placebo-controlled, phase 3 trial. Lancet Oncol. 2020 Jan;21(1):44-59.

21 FDA. FDA approves atezolizumab for PD-L1 positive unresectable locally advanced or metastatic triple-negative breast cancer, 2019. [accessed April 8, 2020]. Available from: https://www.fda.gov/drugs/drug-approvalsand-databases/fda-approves-atezolizumabpd-11-positive-unresectable-locally-advanced-or-metastatic-triple-negative.

22 Clinicaltrials.gov: searched for immunotherapy, Breast Cancer, interventional, not yet recruiting, recruiting, enrolling by invitation, active, Clinicaltrials.gov, 2020. [accessed April 14, 2020]

23 Gao J, Shi LZ, Zhao H, Chen J, Xiong L, He Q, et al. Loss of IFN- $\gamma$ Pathway Genes in Tumor Cells as a Mechanism of Resistance to Anti-CTLA-4 Therapy. Cell. 2016 Oct;167(2):397-404.e9.

24 Zaretsky JM, Garcia-Diaz A, Shin DS, EscuinOrdinas H, Hugo W, Hu-Lieskovan S, et al. Mutations Associated with Acquired Resistance to PD-1 Blockade in Melanoma. N Engl J Med. 2016 Sep;375(9):819-29.
25 Mina LA, Lim S, Bahadur SW, Firoz AT. Immunotherapy for the Treatment of Breast Cancer: Emerging New Data. Breast Cancer (Dove Med Press). 2019 Dec;11:321-8.

26 Zitvogel L, Galluzzi L, Smyth MJ, Kroemer G. Mechanism of action of conventional and targeted anticancer therapies: reinstating immunosurveillance. Immunity. 2013 Jul;39(1):74-88.

27 Konstantinopoulos PA, Waggoner S, Vidal GA, Mita M, Moroney JW, Holloway R, et al. Single-Arm Phases 1 and 2 Trial of Niraparib in Combination With Pembrolizumab in $\mathrm{Pa}$ tients With Recurrent Platinum-Resistant Ovarian Carcinoma. JAMA Oncol. 2019 Jun; 5(8):1141-9.

28 Pellegrino B, Musolino A, Llop-Guevara A, Serra V, De Silva P, Hlavata Z, et al. Homologous Recombination Repair Deficiency and the Immune Response in Breast Cancer: A Literature Review. Transl Oncol. 2020 Feb; 13(2):410-22.

29 Santa-Maria CA, Nanda R. Immune Checkpoint Inhibitor Therapy in Breast Cancer. J Natl Compr Canc Netw. 2018 Oct;16(10): 1259-68.

30 Klein CA. Parallel progression of primary tumours and metastases. Nat Rev Cancer. 2009 Apr;9(4):302-12.

31 Stovgaard ES, Poulsen TS, Balslev E, Nielsen DL, Roslind A, Talman ML, et al. Abstract P504-13: Triple negative breast cancer: Expression of immuno-oncology treatment targets and prevalence of tumor infiltrating lymphocytes in paired primary tumors and metastases. Poster Session Abstracts. Abstracts: 2019 San Antonio Breast Cancer Symposium; December 10-14, 2019; San Antonio, Texas, American Association for Cancer Research, 02152020, P5-04-13-P5-04-13.

32 Keup C, Storbeck M, Hauch S, Hahn P, Sprenger-Haussels M, Tewes M, et al. CellFree DNA Variant Sequencing Using CTCDepleted Blood for Comprehensive Liquid Biopsy Testing in Metastatic Breast Cancer. Cancers (Basel). 2019 Feb;11(2):238.

33 Donini M, Buti S, Lazzarelli S, Bozzetti R, Rivoltini L, Camisaschi C, et al.; GOIRC (Italian Oncology Group for Clinical Research). Dose-finding/phase II trial: bevacizumab, immunotherapy, and chemotherapy (BIC) in metastatic renal cell cancer (mRCC). Antitumor effects and variations of circulating $\mathrm{T}$ regulatory cells (Treg). Target Oncol. 2015 Jun;10(2):277-86.

34 Cai B, Ma P, Ding P, Sun D-W, Bu Q, Zhang $\mathrm{J}$ : Composition and plasticity of triple-negative breast carcinoma-infiltrating regulatory T cells. APMIS DOI: 10.1111/apm.13022.

35 Hossain DM, Panda AK, Manna A, Mohanty $\mathrm{S}$, Bhattacharjee $\mathrm{P}$, Bhattacharyya $\mathrm{S}$, et al. FoxP3 acts as a cotranscription factor with STAT3 in tumor-induced regulatory T cells. Immunity. 2013 Dec;39(6):1057-69.

36 O’Day SJ, Borges VF, Chmielowski B, Rao RD, Abu-Khalaf M, Stopeck A, et al. Abstract CT073: IMPRIME 1 (NCT02981303): A novel phase 2 study in second-line + , metastatic triple negative breast cancer patients shows promising clinical benefit for the combination of the immune checkpoint inhibitor, pembrolizumab (pembro), with the novel innate immune activator, Imprime PGG. AACR virtual meeting I 2020. https://www.abstractsonline.com/pp8/\#!/9045/presentation/10775.

37 Elashi AA, Sasidharan Nair V, Taha RZ Shaath H, Elkord E. DNA methylation of immune checkpoints in the peripheral blood of breast and colorectal cancer patients. OncoImmunology. 2018 Nov;8(2):e1542918.

38 Khalife E, Khodadadi A, Talaeizadeh A, Rahimian L, Nemati M, Jafarzadeh A. Overexpression of Regulatory T Cell-Related Markers (FOXP3, CTLA-4 and GITR) by Peripheral Blood Mononuclear Cells from Patients with Breast Cancer. Asian Pac J Cancer Prev. 2018 Nov;19(11):3019-25.

39 Kawaguchi K, Suzuki E, Yamaguchi A, Yamamoto M, Morita S, Toi M. Altered expression of major immune regulatory molecules in peripheral blood immune cells associated with breast cancer. Breast Cancer. 2017 Jan;24(1): 111-20.

40 Gao H, Kida K, Cohen EN, Alexander A, Lim B, Parker C, et al. Abstract P3-09-12: Peripheral $\mathrm{T}$ cell clonality and exhaustion as novel biomarkers for anti-PD-1 (pembrolizumab) maintenance therapy in patients with metastatic inflammatory breast cancer (mIBC) and non-IBC triple negative breast cancer ( $\mathrm{mT}$ NBC). Poster Session Abstracts. Abstracts: 2019 San Antonio Breast Cancer Symposium; December 10-14, 2019; San Antonio, Texas, American Association for Cancer Research, 02152020, P3-09-12-P3-09-12.

41 Contini P, Ghio M, Poggi A, Filaci G, Indiveri F, Ferrone S, et al. Soluble HLA-A,-B,-C and $-\mathrm{G}$ molecules induce apoptosis in $\mathrm{T}$ and NK CD8+ cells and inhibit cytotoxic T cell activity through CD8 ligation. Eur J Immunol. 2003 Jan;33(1):125-34.

42 Yu DD, Wu Y, Shen HY, Lv MM, Chen WX, Zhang $\mathrm{XH}$, et al. Exosomes in development, metastasis and drug resistance of breast cancer. Cancer Sci. 2015 Aug;106(8):959-64.

43 König L, Kasimir-Bauer S, Hoffmann O, Bittner AK, Wagner B, Manvailer LF, et al. The prognostic impact of soluble and vesicular HLA-G and its relationship to circulating tumor cells in neoadjuvant treated breast cancer patients. Hum Immunol. 2016 Sep;77(9): 791-9.

44 Ugurel S, Rebmann V, Ferrone S, Tilgen W, Grosse-Wilde H, Reinhold U. Soluble human leukocyte antigen-G serum level is elevated in melanoma patients and is further increased by interferon-alpha immunotherapy. Cancer. 2001 Jul;92(2):369-76.

45 Schwich E, Rebmann V, Horn PA, Celik AA, Bade-Döding C, Kimmig R, et al. VesicularBound HLA-G as a Predictive Marker for Disease Progression in Epithelial Ovarian Cancer. Cancers (Basel). 2019 Aug;11(8):11.

46 Chowell D, Morris LG, Grigg CM, Weber JK, Samstein RM, Makarov V, et al. Patient HLA class I genotype influences cancer response to checkpoint blockade immunotherapy. Science. 2018 Feb;359(6375):582-7.

47 McLaughlin J, Han G, Schalper KA, CarvajalHausdorf D, Pelekanou V, Rehman J, et al. Quantitative Assessment of the Heterogeneity of PD-L1 Expression in Non-Small-Cell Lung Cancer. JAMA Oncol. 2016 Jan;2(1): 46-54. 
48 Ilie M, Long-Mira E, Bence C, Butori C, Lassalle S, Bouhlel L, et al. Comparative study of the PD-L1 status between surgically resected specimens and matched biopsies of NSCLC patients reveal major discordances: a potential issue for anti-PD-L1 therapeutic strategies. Ann Oncol. 2016 Jan;27(1):147-53.

49 Guo L, Cao C, Goswami S, Huang X, Ma L, Guo Y, et al. Tumoral PD-1hiCD8+ T cells are partially exhausted and predict favorable outcome in triple-negative breast cancer. Clin Sci (Lond). 2020 Apr;134(7):711-26.

50 Ishiba T, Hoffmann AC, Usher J, Elshimali Y, Sturdevant T, Dang M, et al. Frequencies and expression levels of programmed death ligand 1 (PD-L1) in circulating tumor RNA (ctRNA) in various cancer types. Biochem Biophys Res Commun. 2018 Jun;500(3):621-5.

51 Mazel M, Jacot W, Pantel K, Bartkowiak K, Topart D, Cayrefourcq L, et al. Frequent expression of PD-L1 on circulating breast cancer cells. Mol Oncol. 2015 Nov;9(9):1773-82.

52 Shin DS, Zaretsky JM, Escuin-Ordinas H, Garcia-Diaz A, Hu-Lieskovan S, Kalbasi A, et al. Primary Resistance to PD-1 Blockade Mediated by JAK1/2 Mutations. Cancer Discov. 2017 Feb;7(2):188-201.

53 Janning M, Kobus F, Babayan A, Wikman H, Velthaus JL, Bergmann S, et al. Determination of PD-L1 Expression in Circulating Tumor Cells of NSCLC Patients and Correlation with Response to PD-1/PD-L1 Inhibitors. Cancers (Basel). 2019 Jun;11(6):11.

54 Koh Y, Yagi S, Akamatsu H, Kanai K, Hayata A, Tokudome N, et al. Heterogeneous Expression of Programmed Death Receptor-ligand 1 on Circulating Tumor Cells in Patients With Lung Cancer. Clin Lung Cancer. 2019 Jul; 20(4):270-7.e1.

55 Papadaki MA, Koutsopoulos AV, Tsoulfas PG, Lagoudaki E, Aggouraki D, Monastirioti A, et al. Clinical Relevance of Immune Checkpoints on Circulating Tumor Cells in Breast Cancer. Cancers (Basel). 2020 Feb; 12(2):12.

56 Raimondi C, Carpino G, Nicolazzo C, Gradilone A, Gianni W, Gelibter A, et al. PDL1 and epithelial-mesenchymal transition in circulating tumor cells from non-small cell lung cancer patients: A molecular shield to evade immune system? Oncoimmunology 2017;6:e1315488.

57 Asgarova A, Asgarov K, Godet Y, Peixoto P, Nadaradjane A, Boyer-Guittaut M, Galaine J, Guenat D, Mougey V, Perrard J, Pallandre JR, Bouard A, Balland J, Tirole C, Adotevi O, Hendrick E, Herfs M, Cartron PF, Borg C, Hervouet $\mathrm{E}$ : $\mathrm{PD}-\mathrm{L} 1$ expression is regulated by both DNA methylation and NF-kB during EMT signaling in non-small cell lung carcinoma. Oncoimmunology. 2018; 7(5):e1423170.

58 Schehr JL, Schultz ZD, Warrick JW, Guckenberger DJ, Pezzi HM, Sperger JM, et al. High Specificity in Circulating Tumor Cell Identification Is Required for Accurate Evaluation of Programmed Death-Ligand 1. PLoS One. 2016 Jul;11(7):e0159397.

59 Qu QX, Huang Q, Shen Y, Zhu YB, Zhang $\mathrm{XG}$. The increase of circulating PD-L1-expressing CD68(+) macrophage in ovarian cancer. Tumour Biol. 2016 Apr;37(4):5031-7.
60 Adams DL, Adams DK, He J, Kalhor N, Zhang $\mathrm{M}, \mathrm{Xu} \mathrm{T}$, et al. Sequential Tracking of PD-L1 Expression and RAD50 Induction in Circulating Tumor and Stromal Cells of Lung Cancer Patients Undergoing Radiotherapy. Clin Cancer Res. 2017 Oct;23(19):5948-58.

61 Chen G, Huang AC, Zhang W, Zhang G, Wu $\mathrm{M}, \mathrm{Xu} \mathrm{W}$, et al. Exosomal PD-L1 contributes to immunosuppression and is associated with anti-PD-1 response. Nature. 2018 Aug; 560(7718):382-6.

62 Lux A, Kahlert C, Grützmann R, Pilarsky C. c-Met and PD-L1 on Circulating Exosomes as Diagnostic and Prognostic Markers for Pancreatic Cancer. Int J Mol Sci. 2019 Jul;20(13): 20.

63 Li C, Li C, Zhi C, Liang W, Wang X, Chen X, et al. Clinical significance of PD-L1 expression in serum-derived exosomes in NSCLC patients. J Transl Med. 2019 Oct;17(1):355.

64 Yang Y, Li CW, Chan LC, Wei Y, Hsu JM, Xia W, et al. Exosomal PD-L1 harbors active defense function to suppress $\mathrm{T}$ cell killing of breast cancer cells and promote tumor growth. Cell Res. 2018 Aug;28(8):862-4.

65 Li Y, Cui X, Yang YJ, Chen QQ, Zhong L, Zhang T, et al. Serum sPD-1 and sPD-L1 as Biomarkers for Evaluating the Efficacy of Neoadjuvant Chemotherapy in Triple-Negative Breast Cancer Patients. Clin Breast Cancer. 2019 Oct;19(5):326-332.e1.

66 Buderath P, Schwich E, Jensen C, Horn PA, Kimmig R, Kasimir-Bauer S, et al. Soluble Programmed Death Receptor Ligands sPDL1 and SPD-L2 as Liquid Biopsy Markers for Prognosis and Platinum Response in Epithelial Ovarian Cancer. Front Oncol. 2019 Oct;9: 1015.

67 Schumacher TN, Schreiber RD. Neoantigens in cancer immunotherapy. Science. $2015 \mathrm{Apr}$; 348(6230):69-74.

68 Lewis SM. The mechanism of V(D)J joining: lessons from molecular, immunological, and comparative analyses. Adv Immunol. 1994; $56: 27-150$.

69 Rosati E, Dowds CM, Liaskou E, Henriksen EK, Karlsen TH, Franke A. Overview of methodologies for T-cell receptor repertoire analysis. BMC Biotechnol. 2017 Jul;17(1):61.

70 Gibney GT, Weiner LM, Atkins MB. Predictive biomarkers for checkpoint inhibitorbased immunotherapy. Lancet Oncol. 2016 Dec;17(12):e542-51.

71 Machado JC, Reis J, Fernandes M, Silva R, Cirnes L, Carneiro F, et al. Tumor-specific neoantigens drive $\mathrm{T}$-cell clonotype convergence. Eur J Cancer. 2018;92:S11.

72 Lin KR, Pang DM, Jin YB, Hu Q, Pan YM, Cui $\mathrm{JH}$, et al. Circulating CD8+ T-cell repertoires reveal the biological characteristics of tumors and clinical responses to chemotherapy in breast cancer patients. Cancer Immunol Immunother. 2018 Nov;67(11):1743-52.

73 Page DB, Yuan J, Redmond D, Wen YH, Durack JC, Emerson R, et al. Deep Sequencing of T-cell Receptor DNA as a Biomarker of Clonally Expanded TILs in Breast Cancer after Immunotherapy. Cancer Immunol Res. 2016 Oct;4(10):835-44.

74 Thompson JR, Menon SP. Liquid Biopsies and Cancer Immunotherapy. Cancer J. 2018 Mar/Apr;24(2):78-83.
75 Truesdell J, Miller VA, Fabrizio D. Approach to evaluating tumor mutational burden in routine clinical practice. Transl Lung Cancer Res. 2018 Dec;7(6):678-81.

76 Nguyen A, Garner C, Reddy SK, Sanborn JZ, Benz SC, Seery TE, et al. Three-fold overestimation of tumor mutation burden using 248 gene panel versus whole exome. J Clin Oncol. 2018;36 15_suppl:12117.

77 Yao L, Fu Y, Mohiyuddin M, Lam HYK. ecTMB: a robust method to estimate and classify tumor mutational burden. Sci Rep. 2020 Mar; 10(1):4983.

78 Sadeh R, Fialkoff G, Sharkia I, Rahat A, Nitzan M, Fox-Fisher I, et al. ChIP-seq of plasma cellfree nucleosomes identifies cell-of-origin gene expression programs. Volume 7. 2019.

79 Gandara DR, Paul SM, Kowanetz M, Schleifman E, Zou W, Li Y, et al. Blood-based tumor mutational burden as a predictor of clinical benefit in non-small-cell lung cancer patients treated with atezolizumab. Nat Med. 2018 Sep;24(9):1441-8

80 Koeppel F, Blanchard S, Jovelet C, Genin B, Marcaillou C, Martin E, et al. Whole exome sequencing for determination of tumor mutation load in liquid biopsy from advanced cancer patients. PLoS One. 2017 Nov; 12(11):e0188174.

81 Snyder A, Makarov V, Merghoub T, Yuan J, Zaretsky JM, Desrichard A, et al. Genetic basis for clinical response to CTLA-4 blockade in melanoma. N Engl J Med. 2014 Dec;371(23): 2189-99.

82 Barroso-Sousa R, Jain E, Cohen O, Kim D, Buendia-Buendia J, Winer E, et al. Prevalence and mutational determinants of high tumor mutation burden in breast cancer. Ann Oncol. 2020 Mar;31(3):387-94.

83 Barroso-Sousa R, Keenan TE, Pernas S, Exman $\mathrm{P}$, Jain E, Garrido-Castro AC, et al. Tumor mutational burden and PTEN alterations as molecular correlates of response to PD-1/L1 blockade in metastatic triple-negative breast cancer. Clin Cancer Res. 2020 Jun;26(11):2565-72.

84 Samstein RM, Lee CH, Shoushtari AN, Hellmann MD, Shen R, Janjigian YY, et al. Tumor mutational load predicts survival after immunotherapy across multiple cancer types. Nat Genet. 2019 Feb;51(2):202-6.

85 Yang W, Lee KW, Srivastava RM, Kuo F, Krishna C, Chowell D, et al. Immunogenic neoantigens derived from gene fusions stimulate T cell responses. Nat Med. 2019 May; 25(5):767-75

86 Pierson A, Darrigrand R, Rouillon M, Boulpicante M, Renko ZD, Garcia C, et al. Splicing inhibition enhances the antitumor immune response through increased tumor antigen presentation and altered MHC-I immunopeptidome. bioRxiv. 2019. https://doi. org/10.1101/512681.

87 Xi L, Pham TH, Payabyab EC, Sherry RM, Rosenberg SA, Raffeld M. Circulating Tumor DNA as an Early Indicator of Response to T-cell Transfer Immunotherapy in Metastatic Melanoma. Clin Cancer Res. 2016 Nov;22(22):5480-6.

88 Moding EJ, Liu Y, Nabet BY, Chabon JJ, Chaudhuri AA, Hui AB, et al. Circulating tumor DNA dynamics predict benefit from consolidation immunotherapy in locally advanced non-small-cell lung cancer. Nat Can. 2020;1(2):176-83. 
89 Jensen TJ, Goodman AM, Kato S, Ellison CK, Daniels GA, Kim L, et al. Genome-Wide Sequencing of Cell-Free DNA Identifies Copy-Number Alterations That Can Be Used for Monitoring Response to Immunotherapy in Cancer Patients. Mol Cancer Ther. 2019 Feb;18(2):448-58.

90 Weiss GJ, Beck J, Braun DP, BornemannKolatzki K, Barilla H, Cubello R, et al. Tumor Cell-Free DNA Copy Number Instability Predicts Therapeutic Response to Immunotherapy. Clin Cancer Res. 2017 Sep; 23(17):5074-81.

91 Nolan E, Savas P, Policheni AN, Darcy PK, Vaillant F, Mintoff CP, et al.; Kathleen Cuningham Foundation Consortium for $\mathrm{Re}$ search into Familial Breast Cancer (kConFab). Combined immune checkpoint blockade as a therapeutic strategy for BRCA1-mutated breast cancer. Sci Transl Med. 2017 Jun;9(393):9.

92 Przybytkowski E, Davis T, Hosny A, Eismann J, Matulonis UA, Wulf GM, et al. An immune-centric exploration of BRCA1 and BRCA2 germline mutation related breast and ovarian cancers. BMC Cancer. 2020 Mar;20(1):197.

93 Jiao S, Xia W, Yamaguchi H, Wei Y, Chen MK, Hsu JM, et al. PARP Inhibitor Upregulates PD-L1 Expression and Enhances Cancer-Associated Immunosuppression. Clin Cancer Res. 2017 Jul;23(14):3711-20.

94 Keup C, Storbeck M, Hauch S, Hahn P, Sprenger-Haussels M, Hoffmann O, et al. Multimodal Targeted Deep Sequencing of Circulating Tumor Cells and Matched CellFree DNA Provides a More Comprehensive Tool to Identify Therapeutic Targets in Metastatic Breast Cancer Patients. Cancers (Basel). 2020 Apr;12(5):1084.

95 Keup C, Benyaa K, Hauch S, SprengerHaussels M, Tewes M, Mach P, et al. Targeted deep sequencing revealed variants in cell-free DNA of hormone receptor-positive metastatic breast cancer patients. Cell Mol Life Sci. 2020 Feb;77(3):497-509.

96 Liu Z, Jiang Z, Gao Y, Wang L, Chen C, Wang X. TP53 Mutations Promote Immunogenic Activity in Breast Cancer. J Oncol. 2019 Jun;2019:5952836.

97 Cheng J, Ding X, Xu S, Zhu B, Jia Q. Gene expression profiling identified TP53MutPIK3CAWild as a potential biomarker for patients with triple-negative breast cancer treated with immune checkpoint inhibitors. Oncol Lett. 2020 Apr;19(4):2817-24.

98 Lin E, Cao T, Nagrath S, King MR. Circulating Tumor Cells: Diagnostic and Therapeutic Applications. Annu Rev Biomed Eng. 2018 Jun;20(1):329-52.

99 Mader S, Pantel K. Liquid Biopsy: Current Status and Future Perspectives. Oncol Res Treat. 2017;40(7-8):404-8.

100 Heeke S, Mograbi B, Alix-Panabières C, Hofman P. Never Travel Alone: The Crosstalk of Circulating Tumor Cells and the Blood Microenvironment. Cells. 2019 Jul; 8(7):8.

101 Xue D, Xia T, Wang J, Chong M, Wang S, Zhang C. Role of regulatory $\mathrm{T}$ cells and CD8+ T lymphocytes in the dissemination of circulating tumor cells in primary inva- sive breast cancer. Oncol Lett. 2018 Sep; 16(3):3045-53.

102 Mego M, Gao H, Cohen EN, Anfossi S, Giordano A, Tin S, et al. Circulating tumor cells (CTCs) are associated with abnormalities in peripheral blood dendritic cells in patients with inflammatory breast cancer. Oncotarget. 2017 May;8(22):35656-68.

103 Mego M, Kalavska K, Karaba M, Minarik G, Benca J, Sedlackova T, et al. Abstract P4-01-15: CTC with EMT phenotype are associated with PD-L1 expression in tumor associated stroma in primary breast cancer patients, 2020.

104 Kallergi G, Tsintari V, Sfakianakis S, Bei E, Lagoudaki E, Koutsopoulos A, et al. The prognostic value of JUNB-positive CTCs in metastatic breast cancer: from bioinformatics to phenotypic characterization. Breast Cancer Res. 2019 Aug;21(1):86.

105 Ogorevc E, Kralj-Iglic V, Veranic P. The role of extracellular vesicles in phenotypic cancer transformation. Radiol Oncol. 2013 Jul; 47(3):197-205.

106 Ding G, Zhou L, Qian Y, Fu M, Chen J, Chen J, et al. Pancreatic cancer-derived exosomes transfer miRNAs to dendritic cells and inhibit RFXAP expression via miR-212-3p. Oncotarget. 2015 Oct;6(30):29877-88.

107 Kitai Y, Kawasaki T, Sueyoshi T, Kobiyama K, Ishii KJ, Zou J, et al. DNA-Containing Exosomes Derived from Cancer Cells Treated with Topotecan Activate a STING-Dependent Pathway and Reinforce Antitumor Immunity. J Immunol. 2017 Feb;198(4):1649-59.

108 Ying X, Wu Q, Wu X, Zhu Q, Wang X, Jiang $\mathrm{L}$, et al. Epithelial ovarian cancer-secreted exosomal miR-222-3p induces polarization of tumor-associated macrophages. Oncotarget. 2016 Jul;7(28):43076-87

109 Gabrusiewicz K, Li X, Wei J, Hashimoto Y, Marisetty AL, Ott M, et al. Glioblastoma stem cell-derived exosomes induce M2 macrophages and PD-L1 expression on human monocytes. Oncolmmunology. 2018 Jan; 7(4):e1412909.

110 Zhao J, Schlößer HA, Wang Z, Qin J, Li J, Popp F, et al. Tumor-Derived Extracellular Vesicles Inhibit Natural Killer Cell Function in Pancreatic Cancer. Cancers (Basel). 2019 Jun;11(6):11.

111 Andreola G, Rivoltini L, Castelli C, Huber V, Perego P, Deho P, et al. Induction of lymphocyte apoptosis by tumor cell secretion of FasL-bearing microvesicles. J Exp Med. 2002 May;195(10):1303-16.

112 Poggio M, Hu T, Pai CC, Chu B, Belair CD, Chang A, et al. Suppression of Exosomal PD-L1 Induces Systemic Anti-tumor Immunity and Memory. Cell. 2019 Apr;177(2): 414-27.e13.

113 Wang M, Ji S, Shao G, Zhang J, Zhao K, Wang Z, et al. Effect of exosome biomarkers for diagnosis and prognosis of breast cancer patients. Clin Transl Oncol. 2018 Jul;20(7): 906-11.

114 Kibria G, Ramos EK, Lee KE, Bedoyan S, Huang S, Samaeekia R, et al. A rapid, automated surface protein profiling of single circulating exosomes in human blood. Sci Rep. 2016 Nov;6(1):36502.

115 Li I, Nabet BY. Exosomes in the tumor microenvironment as mediators of cancer therapy resistance. Mol Cancer. 2019 Mar; 18(1):32.

116 Théry C, Ostrowski M, Segura E. Membrane vesicles as conveyors of immune responses. Nat Rev Immunol. 2009 Aug;9(8):581-93.

117 Moroishi T, Hayashi T, Pan WW, Fujita Y, Holt MV, Qin J, et al. The Hippo Pathway Kinases LATS1/2 Suppress Cancer Immunity. Cell. 2016 Dec;167(6):1525-39.e17.

118 Shenoda BB, Ajit SK. Modulation of Immune Responses by Exosomes Derived from Antigen-Presenting Cells. Clin Med Insights Pathol. 2016 Sep;9 Suppl 1:1-8.

119 Raposo G, Nijman HW, Stoorvogel W, Liejendekker R, Harding CV, Melief CJ, et al. B lymphocytes secrete antigen-presenting vesicles. J Exp Med. 1996 Mar;183(3):116172.

120 Vincent-Schneider H, Stumptner-Cuvelette P, Lankar D, Pain S, Raposo G, Benaroch P, et al. Exosomes bearing HLA-DR1 molecules need dendritic cells to efficiently stimulate specific T cells. Int Immunol. $2002 \mathrm{Jul}$; 14(7):713-22.

121 Admyre C, Johansson SM, Paulie S, Gabrielsson S. Direct exosome stimulation of peripheral human $\mathrm{T}$ cells detected by ELISPOT. Eur J Immunol. 2006 Jul;36(7):1772-81.

122 Lindenbergh MF, Stoorvogel W. Antigen Presentation by Extracellular Vesicles from Professional Antigen-Presenting Cells. Annu Rev Immunol. 2018 Apr;36(1):435-59.

123 Tucci M, Passarelli A, Mannavola F, Stucci LS, Ascierto PA, Capone M, et al. Serum exosomes as predictors of clinical response to ipilimumab in metastatic melanoma. OncoImmunology. 2017 Nov;7(2):e1387706.

124 Coticchia CM, Sullivan RJ, Hurley JD, Hu L, Lawrence DP, Flaherty K, et al. Association of plasma exosomal mRNA changes with long-term durable response to ipilimumab (IPI) in metastatic melanoma (MM) patients. J Clin Oncol. 2016;34 15_suppl:3037.

125 Rodríguez M, Silva J, Herrera A, Herrera M, Peña C, Martín P, et al. Exosomes enriched in stemness/metastatic-related mRNAS promote oncogenic potential in breast cancer. Oncotarget. 2015 Dec;6(38):40575-87.

126 Keup C, Mach P, Aktas B, Tewes M, Kolberg HC, Hauch S, et al. RNA Profiles of Circulating Tumor Cells and Extracellular Vesicles for Therapy Stratification of Metastatic Breast Cancer Patients. Clin Chem. 2018 Jul; 64(7):1054-62.

127 König L, Kasimir-Bauer S, Bittner AK, Hoffmann O, Wagner B, Santos Manvailer LF, et al. Elevated levels of extracellular vesicles are associated with therapy failure and disease progression in breast cancer patients undergoing neoadjuvant chemotherapy. OncoImmunology. 2017 Sep;7(1):e1376153.

128 Eichelser C, Stückrath I, Müller V, MildeLangosch K, Wikman H, Pantel K, et al. Increased serum levels of circulating exosomal microRNA-373 in receptor-negative breast cancer patients. Oncotarget. 2014 Oct;5(20): 9650-63.

129 Khan S, Bennit HF, Turay D, Perez M, Mirshahidi S, Yuan Y, et al. Early diagnostic value of survivin and its alternative splice variants in breast cancer. BMC Cancer. 2014 Mar;14(1):176. 